\title{
Microscale methods to assemble mammalian cells into tissue-like structures
}

\author{
GONG PeiYuan ${ }^{1}$, ZHENG WenFu $^{2}$, XIAO Dan $^{1 *} \&$ JIANG XingYu ${ }^{2 *}$ \\ ${ }^{1}$ College of Chemistry, Sichuan University, Chengdu 610064, China; \\ ${ }^{2}$ Key Lab for Biological Effects of Nanomaterials and Nanosafety, National Center for Nanoscience and Technology, Beijing 100190, China
}

Received May 28, 2012; accepted September 24, 2012

\begin{abstract}
Different cell types make up tissues and organs hierarchically and communicate within a complex, three-dimensional (3D) environment. The in vitro recapitulation of tissue-like structures is meaningful, not only for fundamental cell biology research, but also for tissue engineering (TE). Currently, TE research adopts either the top-down or bottom-up approach. The top-down approach involves defining the macroscopic tissue features using biomaterial scaffolds and seeding cells into these scaffolds. Conversely, the bottom-up approach aims at crafting small tissue building blocks with precision-engineered structural and functional microscale features, using physical and/or chemical approaches. The bottom-up strategy takes advantage of the repeating structural and functional units that facilitate cell-cell interactions and cultures multiple cells together as a functional unit of tissue. In this review, we focus on currently available microscale methods that can control mammalian cells to assemble into 3D tissue-like structures.
\end{abstract}

microscale, cell assembly, tissue-like structures, tissue engineering

Citation: Gong P Y, Zheng W F, Xiao D, et al. Microscale methods to assemble mammalian cells into tissue-like structures. Sci China Life Sci, 2012, 55: 862-871, doi: 10.1007/s11427-012-4385-9

The function of a tissue relies upon its constituent cell types and overall organization [1]. Tissue dynamics, including formation, function and regeneration, result from intricate spatiotemporal coordination of numerous individual cells [2], each induced by cell extracellular microenvironment interactions, including the extracellular matrix, neighboring cells and soluble cues. Interactions between the cell and its microenvironment determine its pattern of gene expression and resultant differentiated phenotype despite the fact that the cells share an identical genome. Therefore, cell behavior is largely determined by interactions with the extracellular microenvironment. The spatial orientation and distribution of the extracellular microenvironment directly influences tissue function. Cellular interactions with extracellular matrix molecules and other cells are important issues of fun-

*Corresponding author (email: xiaodan@scu.edu.cn; xingyujiang@ nanoctr.cn) damental cell biology, and play a crucial role in tissue engineering (TE) [3,4]. Proper cell-cell communication through physical contact is crucial for a range of fundamental biological processes and the normal function of tissues. The spatial arrangements of these cellular interactions lead to higher-order function, which is difficult to recapitulate in vitro. Reconstituting functional cell-cell communication in vitro and in three dimension (3D) remains an important challenge in TE.

Current TE research adopts either the top-down or bottom-up approach [5]. The top-down approach involves defining the macroscopic tissue features using biomaterial scaffolds and seeding cells into these scaffolds. These scaffolds can range in size from millimeters to centimeters [6]. This approach has been successful for constructing tissues whose functions are relatively independent from the fine structural features [7]. The major challenge of this approach 
is how to better control the local spatial orientation and distribution of the extracellular microenvironment [8] for engineering complex tissues whose functions are greatly affected by fine structural features. The bottom-up approach takes advantage of repeating structural and functional units, like nephrons, liver lobules, or ganglions $[9,10]$. This approach involves engineering microtissue constructs $[11,12]$ as small tissue building blocks with precision-engineered structural and functional microscale features. The construction of microtissues starts with the basic step of assembling cells into cell clusters to facilitate cell-cell interactions and cultures multiple cells together as functional tissue units. The assembly of cells can be executed via physical and/or chemical methods. Here, we present a comprehensive review of currently available methods that use microscale methods in physics and chemistry to control mammalian cell assembly into tissue-like structures.

\section{Physical methods}

There are several physical methods capable of assembling cells together into small cell clusters to facilitate cell-cell interactions. The principles of these physical methods involve optics, electricity, magnetism, acoustics (Figure 1) and soft lithographic techniques.

\subsection{Laser/optical methods}

Laser/optical methods use a highly focused laser beam to manipulate cells at very high precision [13]. When the laser beam hits the cell, the momentum of the laser beam is transferred to the cell and then causes the cell to be drawn to the center of the beam, thereby manipulating it [14]. Optical tweezers are ideal for single cell manipulation because they are able to manipulate objects with a size of approximately $10 \mu \mathrm{m}$ [15] and exert forces up to hundreds of $\mathrm{pN}$ [16]. The ability of optical tweezers to manipulate cells has been exploited in TE to micropattern different cell types at specific positions, one by one, for the construction of tissue structures $[17,18]$.

Despite the versatility and precision offered by laser methods, drawbacks exist when dealing with cell culture. One critical limiting factors is optical damage. Another is the occurrence of local heating at the focus volume. High intensity at the laser focus point is the main cause of heating [19]. Local heating is a serious issue when manipulating cells, as heat adversely affects enzyme activity and other sensitive cellular functions. To avoid optical damage to cells, a laser-guided direct writing (LGDW) system has been developed, which uses the near-infrared part of the electromagnetic spectrum. LGDW is a variation of the commonly used optical tweezers [20,21] (Figure 1A) and is a unique cellular patterning technique able to deposit cells with micrometer resolution on arbitrary matrices, including soft gels, such as collagen or Matrigel [17,20,22,23]. This has allowed researchers to use the intrinsic ability of endothelial cells to self-assemble into vascular structures [24,25] for the assembly of liver tissue. LGDW is therefore uniquely suited for studying the role of tissue architecture and mechanical properties at the single-cell resolution, and for studying the effects of heterotypic cell-cell interactions un-
A
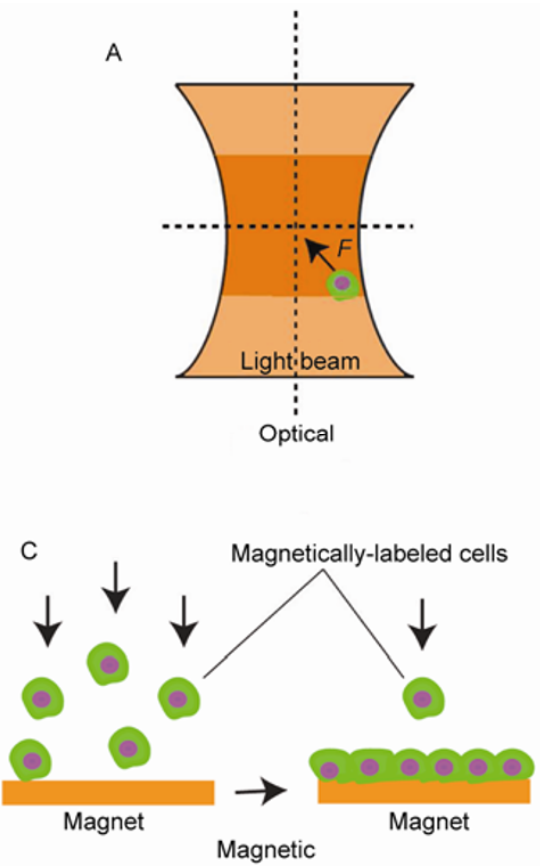

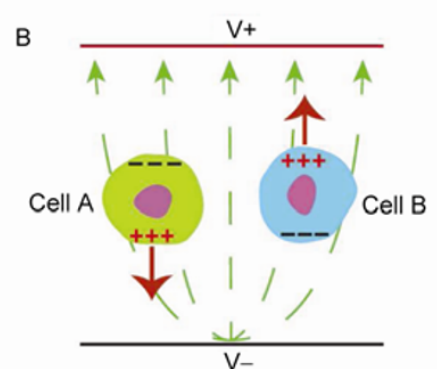

Dielectrophoresis

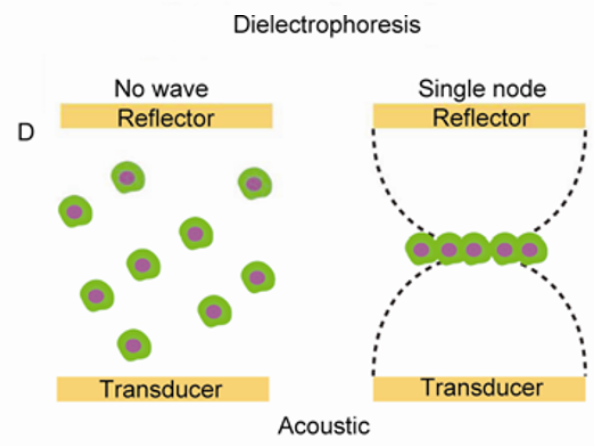

Figure 1 Physical methods of cell assembly. The principle of each method is shown schematically: optics (A), electricity (B), magnetism (C), and acoustics (D). 
derlying processes such as liver morphogenesis, differentiation and angiogenesis [18].

\subsection{Dielectrophoretic methods}

Cells can be manipulated using gradients of an electric field, a phenomenon known as dielectrophoresis (DEP). The DEP force controls movement of the cell to the desired location. Depending on the electrical properties of the cells and media, and the frequency of the electric field, the DEP force acting on the cell can be positive or negative. Movement of cells towards the strong electric field region is termed positive DEP (p-DEP), and movement in the opposite direction is termed negative DEP (n-DEP) (Figure 1B).

Bhatia et al. [26] have reported a method for the rapid formation of reproducible, high-resolution 3D cellular structures within a photopolymerizable hydrogel using p-DEP forces. The DEP cell patterning chamber sandwiched a suspension of cells in un-cross-linked prepolymer solution between two conductive indium tin oxide (ITO)-coated glass slides. On the bottom slide, a thin micropatterned layer of insulating photo epoxy masked most of the conductive surface to form electrode arrays in all remaining uninsulated areas. An alternating current bias applied across the top and bottom slides produced a spatially nonuniform electric field that was strongest near the gaps in the dielectric layer. The p-DEP forces propelled cells toward locations of high electric field strength. Using this techniques, Bhatia et al. demonstrated parallel formation of cell clusters of precise size and shape within the thin hydrogel. By modulating cell-cell interactions in 3D clusters, they also demonstrated that microscale tissue organization regulates bovine articular chondrocyte biosynthesis.

Matsue et al. [27] have reported a method for patterning different cell types based on n-DEP, without any special pretreatment of culture slides. An interdigitated array (IDA) electrode with four independent microelectrode subunits, was fabricated with ITO and used as a template to form cellular micropatterns. A suspension of the first cell type was introduced into the patterning device between the upper IDA and bottom slide. In the system, the n-DEP force directs cells toward a weaker region of electric field strength. The cells aligned above one IDA band, since the aligned areas on the slide were regions with a lower electric field. After removing excess cells, the second cell type was patterned in lines using the same method. Periodic and alternate cell lines incorporating two cell types were fabricated by changing the alternating current voltage mode.

There are two main problems associated with manipulating cells using an electric field: (i) the Joule heating effect produces large temperature gradients in the medium which can affect cell viability; (ii) the influence of the electric field on cell physiology [28], such as voltage-sensitive proteins [29]. Lowering the electric potential and shortening the manipulation time may be a solution for these problems.

\subsection{Magnetic methods}

Another cell manipulation method uses a magnetic field $[30,31]$. In this method, magnetic particles usually have a magnetic core and a coating tailored to bind to specific molecules, so magnetic particles could are selectively attached to cells for TE applications [32]. Cells labeled with magnetic nanoparticles can then be remotely manipulated by applying a magnetic field [33] (Figure 1C).

Slater et al. [34] have used a magnetic method to form the arrangement of multicellular spheroids. They labeled cell membrane proteins with biotin and then linked streptavidin paramagnetic particles onto the biotinylated cell surface. Magnetically labeled cells were cultured in hanging drops to generate magnetic spheroids. The hanging drop method for growing multicellular spheroids is simple and could provide a substrate-free route for obtaining spheroids with a narrow size distribution. The magnetic spheroids were patterned using magnetic fields, and then fused together to form a larger tissue construct.

Kamihira et al. [35] have reported a method for the fabrication of cell sheets using magnetite cationic liposomes (MCLs) and a combination of two magnetic force-based techniques, magnetofection and magnetic cell accumulation. A retroviral vector, encoding an expression cassette of vascular endothelial growth factor (VEGF), was labeled with MCLs to magnetically attract the particles onto a monolayer of mouse myoblast cells for gene delivery. During fabrication of the tissue constructs, MCL-labeled cells accumulated in the presence of a magnetic field to promote spontaneous formation of a multilayered cell sheet. These VEGF gene-engineered cell sheets were then subcutaneously implanted into nude mice, where they produced well vascularized tissues with substantial mass, thickness and cell density.

Alsberg et al. [36] have reported an approach for magnetically driven organization of ordered cellular structures, without using microfabricated substrates, potentially harmful electrical fields, or physical attachment to or uptake of material by the cells. Inert, biologically compatible ferrofluids, consisting of suspensions of protein-passivated iron oxide nanoparticles, drove cell organization into linear structures in the magnetic field. The purpose of the magnetic nanoparticles was to induce an effective magnetization in the extracellular fluid in order to guide the cells into highly organized structures. The linear cell structures are stable and can be further cultured without the magnetic field and nanoparticles.

McDevitt et al. [37] have reported a simple, physical entrapment of magnetic microparticles (MagMPs) within the extracellular space of stem cell spheroids. MagMPs can be efficiently and stably incorporated, in a dose-dependent manner, into the extracellular environment of 3D stem cell aggregates, without the need for biochemical modification of the cell membrane or material adhesive properties. 
MagMP entrapment within multicellular aggregates enables directed movement and assembly of multicellular spheroids in suspension culture, with an externally applied magnetic field, to yield complex organization of 3D constructs.

\subsection{Acoustic methods}

Another method for contactless cell manipulation is ultrasonic waves. Ultrasonic transducers generate waves, which subject cells to a mechanical force dependent on the cell volume and frequency of the ultrasonic waves. Cells can be concentrated in either the nodes or antinodes of the periodic wave pattern $[13,38]$. The ultrasonic waves employed here are not detrimental to cell viability [39].

Wiklund et al. [40] have demonstrated 3D caging, enrichment and shape-specific aggregation, combined with high resolution imaging of cells in an ultrasonically actuated microfluidic chip. It is possible to construct the trapped cells as either a $2 \mathrm{D}$ monolayer or a compact $3 \mathrm{D}$ aggregate. This is because the cage is simultaneously actuated at two frequencies, the dimension of which can be optimized for individual cell handling, or handling of aggregates containing up to a few hundred cells. Transformation between monolayer and multilayer structures is interesting for the investigation of cell-cell interactions, with control of the number of neighbors for each cell.

Huang et al. [41] have developed an active patterning technique, named "acoustic tweezers", that uses standing surface acoustic waves (SSAW) to manipulate and pattern cells in the microfluidic chip (Figure 1D). A surface acoustic wave (SAW) is a sound wave that propagates along the surface of an elastic material. This technique does not require pre-treatment of the substrate or cells and is applicable to any type of cell, regardless of size, shape, or electrical/magnetic/optical properties. Furthermore, its power intensity is lower than that of optical tweezers. With advantages in versatility, miniaturization, power consumption, speed and technical simplicity, "acoustic tweezers" are a powerful tool for many applications including TE.

\subsection{Soft lithographic techniques}

Microfabrication has been a useful tool in biological applications, partly because of the emergence of soft lithography to fabricate microscale devices without the use of expensive photolithographic equipment [42]. Soft lithography is a set of microfabrication techniques, mainly comprising microcontact printing, microtransfer molding and molding in microchannels [43,44]. These techniques use elastomeric stamps, for example polydimethylsiloxane (PDMS), fabricated from patterned silicon wafers to print or mold materials at resolutions as low as several hundred nanometers [44-47]. Therefore, soft lithography can be used to control the topography and spatial distribution of molecules on a surface, as well as the subsequent deposition of cells [48,49].
Soft lithographic methods can also be used to fabricate microfluidic channels for TE in a convenient, rapid and inexpensive manner [50].

The self-assembled monolayer (SAM) of alkanethiol can be reductively desorbed from the gold substrate by application of a negative electrical potential. If cells are cultured on the alkanethiol SAM, which is made adhesive by covalent bonding of Arg-Gly-Asp (RGD)-peptides to the alkanethiol molecules, the electrochemical desorption of SAM results in detachment of the cell sheets from the gold surface in a reliable and rapid manner. Fukuda et al. applied microfabrication and microcontact printing with alkanethiol SAMs for engineering and harvesting cell sheets and spheroids in vitro [51]. The detached cell sheets could be easily attached to other cell sheets in succession to form a multilayered cell sheet.

Takeuchi et al. have engineered a 3D tissue with a neuronal network that can be easily manipulated and transplanted onto the host brain tissue in vivo [52]. A PDMS microchamber array facilitated formation of multiple neurospheroids, which in turn interconnected via neuronal processes to form a centimeter-sized neurospheroid network (NSN). They also developed a simple technique for transplanting NSN onto specific brain areas at a high transfer rate, termed the NSN-stamping method.

Recently, several groups have used shape-changing membranes to fabricate tubes with different materials and sizes for biological applications, such as a platform for cell culturing $[53,54]$. We have reported a general strategy to fabricate tubular structures with multiple cell types as different layers of the tube walls [55]. By using a stress-induced rolling membrane (SIRM) technique, tubes with controllable sizes, which are supported by the balance of the internal stress and strain, form very quickly after release. By rolling, simple patterns on 2D membranes are transformed into complex patterns in 3D tubes (Figure 2). These tubes could mimic blood vessels in which different types of cells constitute different layers of the tubular wall. Therefore, this strategy provides a new perspective to precisely pattern different types of cells into 3D structures: first patterning different cells on a 2D surface, which could undergo changes in shape, and then deforming the $2 \mathrm{D}$ surface into a 3D structure.

\section{Chemical methods}

Construction of 3D multicellular tissues requires directed assembly of cells. Current approaches to control 3D cell assembly rely on intrinsic cell adhesive properties or engineering cellular interactions. Intercellular adhesion is mediated through natural cell-cell adhesion mechanisms, such as cadherins [56,57]. Therefore, cell-surface engineering methodologies are an alternative way to promote and stabilize multicellular assembly. Functional molecules can be 

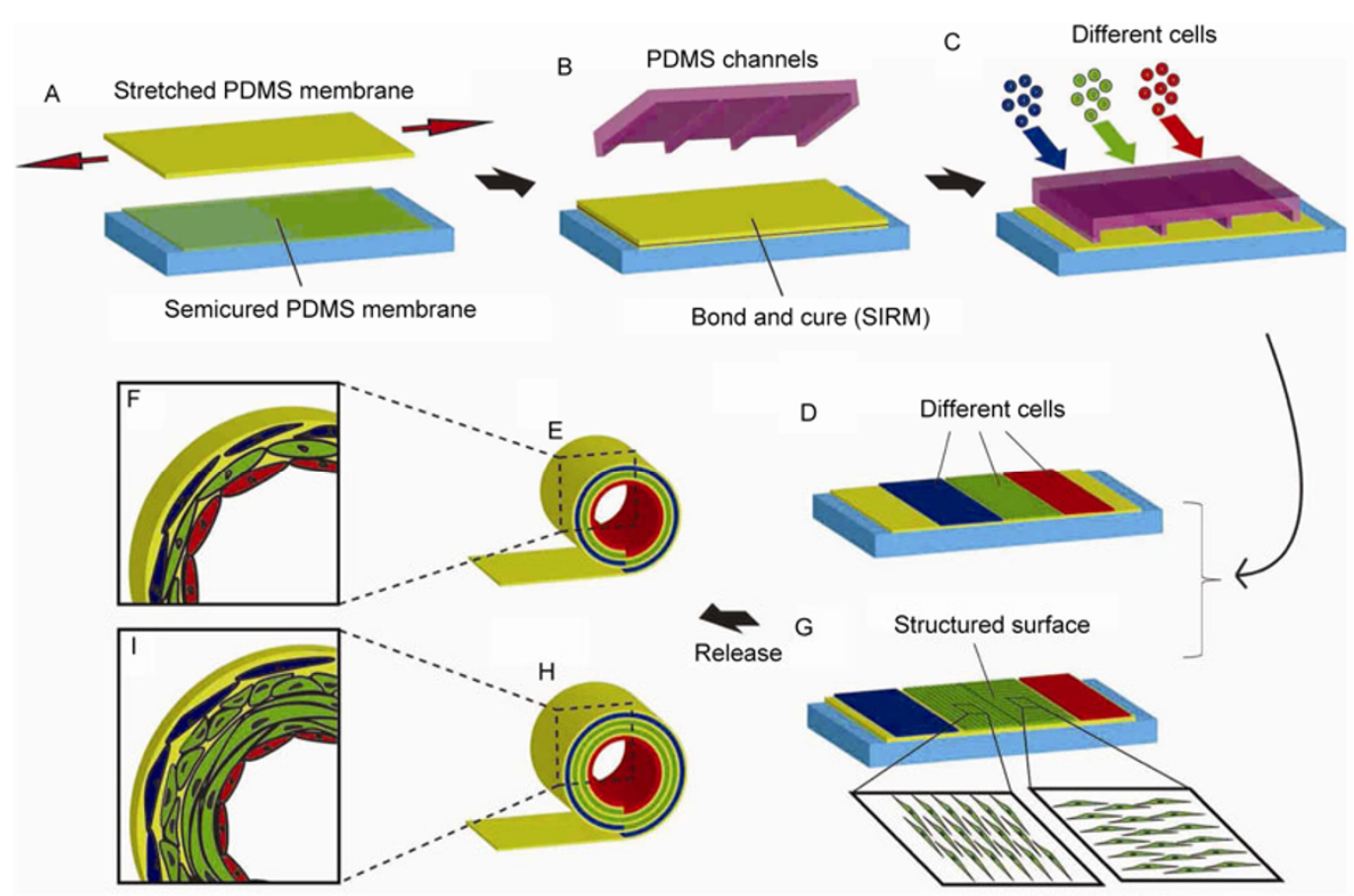

Aligned SMCs

Figure 2 Schematic illustration of a stress-induced rolling membrane (SIRM) and tubes with multiple cell types within the walls [55]. A thin PDMS membrane is stretched as the top layer of the SIRM to cover a semicured PDMS membrane (A). After curing the two layers to cause adhesion (B), microfluidic channels cover the SIRM surface with channel direction being vertical to the stretching direction (C). Different cells are delivered via microfluidic channels to the SIRM surface. Once the cells attach to the SIRM surface, the microchannels are removed (D). One end of the SIRM is released by cutting its edge. The SIRM rolls up into a tube and each cell type is delivered to a designated position with the tubular wall ( $\mathrm{E}$ and $\mathrm{F}$ ). Cell alignment is controlled by contact guidance on structured surfaces (microgrooves, G). After release, cell orientation inside the tubes is either circumferential or longitudinal (H and I).

introduced onto cell membranes to construct multicellular tissues through the interactions between different functional groups on the modified surfaces. The cell membrane of mammalian cells is a complex composite of lipids, proteins and carbohydrates [58]. The lipid bilayer structure forms the barrier between the cell cytoplasm and its environment. The protein and carbohydrate components introduce selective interaction sites. Most cell-surface engineering methodologies take advantage of these cell membrane components (Figure 3). Use of antibodies, aptamers, or other engineered binding molecules for cell surface attachment, allows subsequent attachment of cells. Each of these approaches finds utility in different situations [59].

\subsection{Chemical cell surface modification}

The most straightforward strategy to assembly cells perhaps, is chemical conjugation of molecules to the cells via pre-existing functional groups on the cell surface. However, this provides the least control over the resulting cell surface remodeling. Functional groups naturally present on the cell surface as part of proteins or carbohydrates and are appealing docking sites for covalent conjugation of functional materials to cells, since they do not require any chemical cell preconditioning. The most common approach involves direct chemical reaction of an amino (lysine- $\mathrm{NH}_{2}$ ) or thiol
(cysteine-SH) group presented on a cell membrane protein, with a compatible reactive group on exogenous molecules, polymers, or functional materials (Figure 3A).

Biotinylation of cell surfaces is most frequently used through reaction of primary amine groups on the cell membrane with amine-reactive biotin, such as N-hydroxy- succinimide biotin derivatives (NHS-biotin). Once a cell is labeled with biotin, it can be readily functionalized with a wide range of biotinylated molecules through the biotin-avidin interaction [60,61] (Figure 3B). Sakai et al. [62] applied a rapid cell-cell adhesion method for construction of heterospheroids with homogenous distribution of different cell types, using NHS-biotin derivatives through the biotin-avidin interaction. Heath et al. [63] also reported that microfluidic-guided flow-patterning of surfaces could be an avenue for the construction of tissues with architectural precision at the single cell level. In their approach, cell membrane proteins were biotinylated by reacting with NHSbiotin, then addressed via cysteine-engineered streptavidin-conjugated oligonucleotides. The cells were then serially dispensed onto an oligonucleotide-patterned slide, where each individual cell located to a single cognate spot.

Thiol-reactive functional groups, such as maleimide, can be employed to link molecules to cell surfaces [64]. Other reactive groups of aldehydes and ketones can be generated through chemical or enzymatic treatment of existing cell 


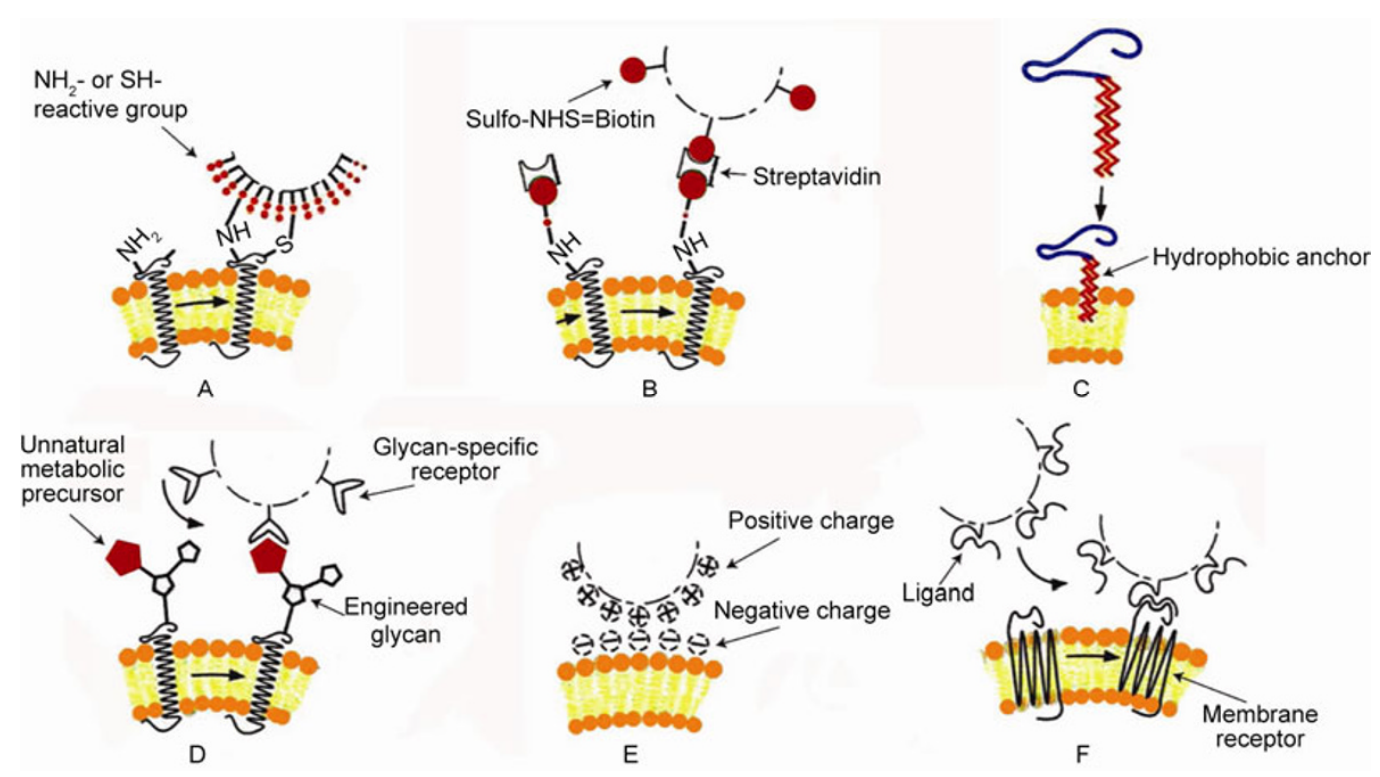

Figure 3 Schematic of the bioengineering methodologies to modify the surface of live mammalian cells.

surface carbohydrates, and have also been used successfully to covalently attach surface-modifying molecules. For example, Shakesheff et al. [65] generated aldehyde groups on the cell surface through the reaction of selective periodate oxidation of sialic acid residues, which selectively ligate biotin hydrazide to the cell surface. The biotinylated cells then crosslinked with avidin, resulting in rapid artificial aggregation of the cells. Based on this method, they also generated layered heterotypic 3D aggregates using free surface avidin groups on a homotypic aggregate to selectively attach a second cell type [66].

\subsection{Hydrophobic insertion}

Integral membrane proteins are anchored into the cell membrane through hydrophobic residues in their transmembrane helices, which make complementary interactions with the hydrophobic lipid bilayer [67]. This hydrophobic effect governing incorporation and orientation of transmembrane proteins can also be used for cell surface engineering. When molecules, in which the functional groups are conjugated with an appropriate hydrophobic anchor, are combined with cultured cells, the hydrophobic moiety can spontaneously insert into the lipid bilayer, leaving the functional groups anchored to and exposed on the cell surface (Figure 3C).

Iwata et al. [68] proposed a new method for the microencapsulation of islets with a layer of living cells using amphiphilic polyethylene glycol (PEG), biotin-PEG-lipid and biotin-streptavidin reaction. Biotin molecules were introduced on the cell surface after incorporation of biotin-PEG-lipid into the phospholipid bilayer membrane by hydrophobic interaction. Then, streptavidin was immobilized onto the biotin-PEG-lipid-modified surface. Unoccu- pied streptavidin sites were available for cell-immobilization because streptavidin has four binding sites for biotin. Islets were also treated with biotin-PEG-lipid. Streptavidin-coated cells were immobilized at the surface of the islets by reacting streptavidin-coated cells with biotin-modified islets. They also employed DNA hybridization instead of the biotin-streptavidin interaction [69]. Polyadenine (poly A) and polythymine (poly T) were introduced onto the surfaces of the islets and cells respectively. The cells were thereby immobilized on the surface of the islets through hybridization of poly $\mathrm{A}$ and poly $\mathrm{T}$ to form a layer of living cells on the surface of the islets. This strategy has also succeeded in the cell-cell attachment induced by hybridization of DNA-conjugated PEG-lipid [70].

Yousaf et al. [71] have developed a strategy to induce specific and stable 3D cell-cell contacts through chemoselective cell-surface engineering, based on liposome delivery and fusion, to display bio-orthogonal functional groups from cell membranes. This strategy used tailored liposomes to modify living cell surfaces through membrane fusion for the delivery of ketone or oxyamine groups to different cell populations. 3D tissue-like structures were generated through the chemoselective oxime ligation of the bio-orthogonal ketone and oxyamine molecules presented on the cell surfaces (Figure 4).

\subsection{Metabolic or genetic introduction}

Metabolic introduction of chemical functional groups, that are absent from the native plasma membrane, is an alternative strategy for engineering cell surfaces [72] (Figure 3D). Certain enzymes involved in the biosynthesis of cell surface molecules are tolerant to a degree of structural variability in their substrates. This means cells can be incubated with 

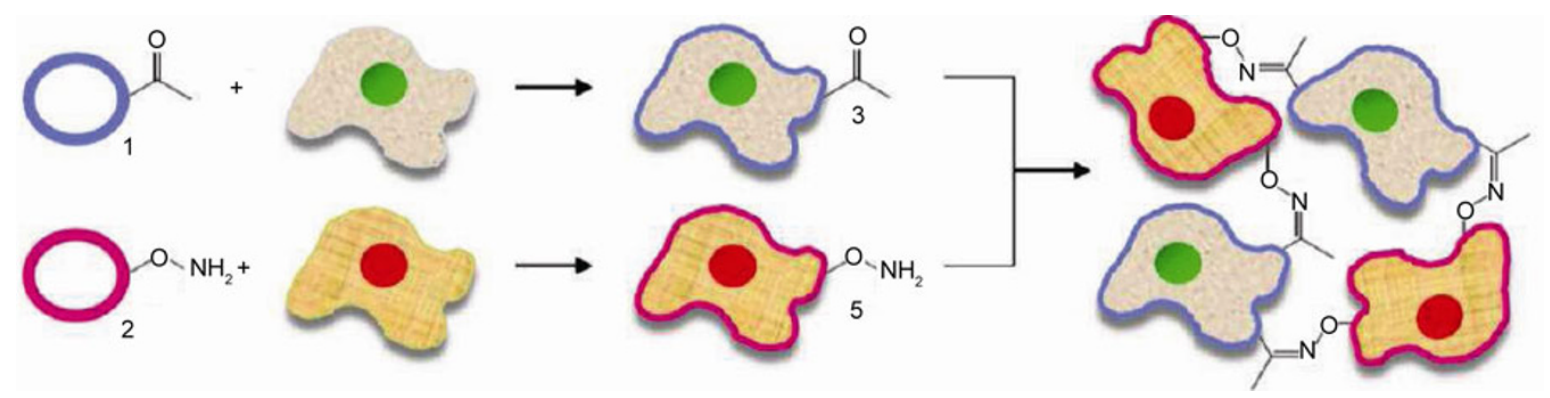

Figure 4 Schematic illustration of 3D spheroid formation via liposome fusion and chemoselective cell-surface tailoring [71]. Two populations of cells were cultured separately with ketone- or oxyamine-containing liposomes, resulting in membrane fusion and subsequent tethering of ketones and oxyamines

from the cell surface. Upon mixing these cell populations, clustering and tissue-like formation occurred based on chemoselective oxime conjugation.

nonphysiological amino sugar analogues, which are taken up and metabolized, resulting in the incorporation of glycoconjugates on the exterior of the cell through natural carbohydrate biosynthetic pathways. In particular, sialic acid is an appealing target of metabolic cell-surface engineering for generating molecular handles [73-75]. As the most abundant terminal components of membrane glycolipids, they are positioned on the outer periphery of the cell, and are therefore situated in ideal proximity for subsequent attachment of the compatible reactive molecules [76-78]. The same concept can be applied to metabolically install reactive thiol groups into the outer periphery of the glycocalyx where they are most accessible to maleimide-derivatized materials [79].

Recently, Gartner and Bertozzi [80] reported bottom-up synthesis of microtissues composed of multiple cell types with programmed connectivity. Cells were incubated with $\mathrm{N}$-azidoacetylmannosamine, resulting in the incorporation of azido sialic acid residues on the cell surface. These residues were subsequently reacted by Staudinger ligation with phosphine-conjugated ssDNA, or by copper-free click chemistry with difluorinated cyclooctyne-conjugated ssDNA, to permit specific adhesive properties. Hybridization between complementary DNA-coated cells enabled assembly of multicellular structures with defined cell-cell contacts (Figure 5). They demonstrated that the assembly process depends on DNA sequence complexity, density and total cell concentration. Thus, cell assembly can be highly controlled. They also used this strategy to construct a paracrine signaling network in isolated 3D microtissues.

Genetic engineering can also be used to introduce bio-orthogonal reactive groups on the cell surface, creating sites for selective modification of cells via a combination of traditional molecular biology and exogenous materials science/chemical methods [81,82]. Although this approach requires genetic manipulation of the substrate cell, it allows the location and identity of the reactive site on the cell surface to be specified, providing greater control over the nature of subsequent cell surface remodeling than linkages introduced through naturally occurring reactive groups described above.

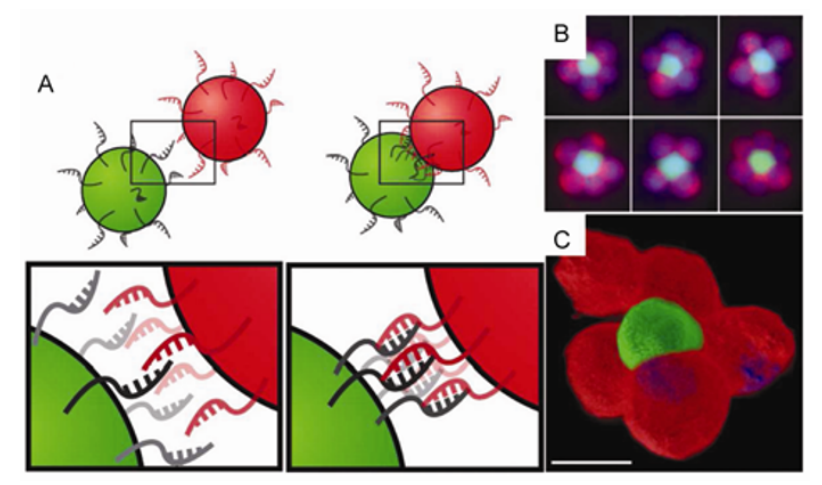

Figure 5 Oligonucleotides direct the synthesis of 3D multicellular structures with defined patterns of connectivity [80]. A, Cells bearing complementary cell surface oligonucleotides react to form stable cell-cell contacts. $\mathrm{B}$, Discrete multicellular structures at higher magnification. C, 3D reconstruction of a single multicellular structure encapsulated in agarose using deconvolution fluorescence microscopy. Scale bar, $10 \mu \mathrm{m}$.

\subsection{Electrostatic interactions}

The surface of mammalian cells carries a net negative charge as a result of phosphate groups of phospholipids, carboxylate groups on proteins and sialic acids terminating glycoprotein sugar chains. The high ionic strength of physiological solutions makes monovalent electrostatic interactions with cells very weak, but polymers or nanoparticles with many cationic sites can bind stably to cells via multivalent electrostatic interactions (Figure 3E).

Rajagopalan et al. [83] demonstrated a bilayer structure composed of hepatocytes and other cells by preparing a polyelectrolyte (PE) multilayer consisting of chitosan and DNA on the hepatocyte surface. Chitosan and DNA were incorporated as the cationic and anionic PEs respectively, because the two kinds of molecules are ionic at $\mathrm{pH}$ values compatible for cell culture. They assembled ultrathin polymer scaffolds on top of a confluent cell monolayer by sequential deposition of oppositely charged PEs. This maintained the morphology, cytoskeletal structure, and liver-specific functions of hepatocytes in vitro, and provided a cell-adhesive surface on which a second layer of cells was cultured, resulting in layered architectures. However, chi- 
tosan cannot dissolve in neutral buffer solutions and fabrication of polyelectrolyte multilayers onto the cell surface is limited owing to the cytotoxicity of polycations $[84,85]$.

\subsection{Ligand-receptor interactions}

Physiologically expressed transmembrane receptors are tempting targets to conjugate biomaterials functionalized with their respective ligands to cells. However, receptor-ligand interactions are transient in nature, determined by intrinsic binding and dissociation kinetics [86], which restricts their utility for stable coupling of material-cell surfaces [87]. However, multivalent binding of ligand-decorated materials with cell surface receptors can lead to stable cell surface binding [88,89] (Figure 3F).

Fibronectin (FN) is a flexible multifunctional glycoprotein that plays an important role in cell attachment [90]. FN can interact not only with a variety of ECM proteins, such as collagens (gelatins) and glycosaminoglycans, but also with its integrin cell surface receptor [91]. Although FN and gelatin have a negative charge under physiological conditions, they interact with each other because FN has a collagen binding domain. Akashi et al. prepared the FN-gelatin (FN-G) nanofilms on the surface of the first cell layer, providing a suitable cell adhesive surface similar to natural ECM for the second cell layer [92]. Consequently, fourlayered architectures of fibroblast cells were fabricated based on the appropriate thickness of FN-G nanofilms. They also developed a simple and rapid bottom-up approach, called the cell-accumulation technique, by single cell coating using FN-G nanofilms [93]. Since FN-G nanofilms prepared on individual cell surfaces provide an interactive property with the integrin receptor of the cell membrane, cell-cell adhesion of all seeded cells in 3D can be induced at the same time. This approach provided approximately $8 \mathrm{~L}$ of 3D tissues after only one day of incubation through FN-G nanofilm fabrication, and the layer number, cell type and location were all successfully controlled by altering the seeding cell number and order.

\section{Conclusion and perspectives}

Microscale methods for TE have generated much interest. The methods for assembly of cells involving physical and chemical approaches allow cells to form 3D microstructures. These methods can recapitulate the extracellular microenvironment around the cells for engineering microtissue constructs.

The major challenge we presently face is the reconstruction of tissue-like structures that function as native tissues in the body. We are still far from this goal so far. We need to precisely control cell-cell and cell layer-cell layer interactions to fabricate multi-cellular assemblies with structural integrity and function. Successful cases indicate that the adequate combination of different techniques can address these problems. In many previous studies, physical methods have been used in conjunction with chemical methods to construct useful structures. The bottom-up and top-down approaches also need close coupling with each other to enrich cell manipulation methods in TE. This would be possible by either multiplexing bottom-up microtissues or by incorporating bottom-up control features in top-down scaffolds.

Another challenge is the biodegradability and compatibility of biomaterials used in in vitro tissue construction. How to improve this field needs close cooperation between chemists, biologists and material scientists.

Overall, with the emergence of new technologies and the improvement of present methods, the prospect of cell-assembly in TE is promising.

This work was supported by Ministry of Science and Technology of China (Grant Nos. 2009CB930001 and 2011CB933201), Chinese Academy of Sciences (Grant No. KJCX2-YW-M15), and the National Natural Science Foundation of China (Grant Nos. 20890020, 90813032, 21025520 and 51073045).

1 Nelson C M, Bissell M J. Of extracellular matrix, scaffolds, and signaling: tissue architecture regulates development, homeostasis, and cancer. Annu Rev Cell Dev Biol, 2006, 22: 287-309

2 Kleinman H K, Philp D, Hoffman M P. Role of the extracellular matrix in morphogenesis. Curr Opin Biotechnol, 2003, 14: 526-532

3 Meshel A S, Wei Q, Adelstein R S, et al. Basic mechanism of three-dimensional collagen fibre transport by fibroblasts. Nat Cell Biol, 2005, 7: 157-164

4 Isenberg B C, Williams C, Tranquillo R T. Endothelialization and flow conditioning of fibrin-based media-equivalents. Ann Biomed Eng, 2006, 34: 971-985

5 Choudhury D, Mo X, Iliescu C, et al. Exploitation of physical and chemical constraints for three-dimensional microtissue construction in microfluidics. Biomicrofluidics, 2011, 5: 022203

6 Hollister S J. Porous scaffold design for tissue engineering. Nat Mater, 2006, 5: 590-590

7 Hutmacher D W. Scaffolds in tissue engineering bone and cartilage. Biomaterials, 2000, 21: 2529-2543

8 Causa F, Netti P A, Ambrosio L. A multi-functional scaffold for tissue regeneration: The need to engineer a tissue analogue. Biomaterials, 2007, 28: 5093-5099

9 Du Y, Lo E, Ali S, et al. Directed assembly of cell-laden microgels for fabrication of 3D tissue constructs. Proc Natl Acad Sci USA, 2008, 105: 9522-9527

10 McGuigan A P, Sefton M V. Vascularized organoid engineered by modular assembly enables blood perfusion. Proc Natl Acad Sci USA, 2006, 103: 11461-11466

11 Khademhosseini A, Langer R. Microengineered hydrogels for tissue engineering. Biomaterials, 2007, 28: 5087-5092

12 Nichol J W, Khademhosseini A. Modular tissue engineering: engineering biological tissues from the bottom up. Soft Matter, 2009, 5: $1312-1319$

13 Nilsson J, Evander M, Hammarström B, et al. Review of cell and particle trapping in microfluidic systems. Anal Chim Acta, 2009, 649: 141-157

14 Molloy J E, Padgett M J. Lights, action: optical tweezers. Contemp Phys, 2002, 43: 241-258

15 Ashkin A, Dziedzic J. Optical trapping and manipulation of viruses and bacteria. Science, 1987, 235: 1517-1520

16 Piggee C. Optical tweezers: not just for physicists anymore. Anal 
Chem, 2008, 81: 16-19

17 Nahmias Y, Schwartz R E, Verfaillie C M, et al. Laser-guided direct writing for three-dimensional tissue engineering. Biotechnol Bioeng, 2005, 92: 129-136

18 Nahmias Y, Odde D J. Micropatterning of living cells by laser-guided direct writing: application to fabrication of hepatic-endothelial sinusoid-like structures. Nat Protoc, 2006, 1: 2288-2296

19 Neuman K C, Nagy A. Single-molecule force spectroscopy: optical tweezers, magnetic tweezers and atomic force microscopy. Nat Methods, 2008, 5: 491-505

20 Nahmias Y K, Gao B Z, Odde D J. Dimensionless parameters for the design of optical traps and laser guidance systems. Appl Optics, 2004, 43: 3999-4006

21 Nahmias Y K, Odde D J. Analysis of radiation forces in laser trapping and laser-guided direct writing applications. IEEE J Quantum Electron, 2002, 38: 131-141

22 Odde D J, Renn M J. Laser-guided direct writing for applications in biotechnology. Trends Biotechnol, 1999, 17: 385-389

23 Odde D J, Renn M J. Laser-guided direct writing of living cells. Biotechnol Bioeng, 2000, 67: 312-318

24 Kubota Y, Kleinman H K, Martin G R, et al. Role of laminin and basement membrane in the morphological differentiation of human endothelial cells into capillary-like structures. J Cell Biol, 1988, 107: 1589-1598

25 Vernon R B, Angello J C, Iruelaarispe M L, et al. Reorganization of basement membrane matrices by cellular traction promotes the formation of cellular networks in vitro. Lab Invest, 1992, 66: 536-547

26 Albrecht D R, Underhill G H, Wassermann T B, et al. Probing the role of multicellular organization in three-dimensional microenvironments. Nat Methods, 2006, 3: 369-375

27 Suzuki M, Yasukawa T, Shiku H, et al. Negative dielectrophoretic patterning with different cell types. Biosens Bioelectron, 2008, 24: 1043-1047

28 Voldman J. Electrical forces for microscale cell manipulation. Annu Rev Biomed Eng, 2006, 8: 425-454

29 Catterall A W. Structure and function of voltage-gated ion channels. Annu Rev Biochem, 1995, 64: 493-531

30 Winkleman A, Gudiksen K L, Ryan D, et al. A magnetic trap for living cells suspended in a paramagnetic buffer. Appl Phys Lett, 2004, 85: 2411-2413

31 Kimura T, Yamato M, Nara A. Particle trapping and undulation of a liquid surface using a microscopically modulated magnetic field. Langmuir, 2004, 20: 572-574

32 Ino K, Okochi M, Konishi N, et al. Cell culture arrays using magnetic force-based cell patterning for dynamic single cell analysis. Lab Chip, 2008, 8: 134-142

33 Dobson J. Remote control of cellular behaviour with magnetic nanoparticles. Nat Nanotechnol, 2008, 3: 139-143

34 Ho V H B, Muller K H, Barcza A, et al. Generation and manipulation of magnetic multicellular spheroids. Biomaterials, 2010, 31: 3095-3102

35 Akiyama H, Ito A, Kawabe $\mathrm{Y}$, et al. Genetically engineered angiogenic cell sheets using magnetic force-based gene delivery and tissue fabrication techniques. Biomaterials, 2010, 31: 1251-1259

36 Krebs M D, Erb R M, Yellen B B, et al. Formation of ordered cellular structures in suspension via label-free negative magnetophoresis. Nano Lett, 2009, 9: 1812-1817

37 Bratt-Leal A M, Kepple K L, Carpenedo R L, et al. Magnetic manipulation and spatial patterning of multi-cellular stem cell aggregates. Integr Biol, 2011, 3: 1224-1232

38 Laurell T, Petersson F, Nilsson A. Chip integrated strategies for acoustic separation and manipulation of cells and particles. Chem Soc Rev, 2007, 36: 492-506

39 Hultström J, Manneberg O, Dopf K, et al. Proliferation and viability of adherent cells manipulated by standing-wave ultrasound in a microfluidic chip. Ultrasound Med Biol, 2007, 33: 145-151

40 Manneberg O, Vanherberghen B, Svennebring J, et al. A threedimensional ultrasonic cage for characterization of individual cells.
Appl Phys Lett, 2008, 93: 063901

41 Shi J, Ahmed D, Mao X, et al. Acoustic tweezers: patterning cells and microparticles using standing surface acoustic waves (SSAW). Lab Chip, 2009, 9: 2890-2895

42 Whitesides G M, Ostuni E, Takayama S, et al. Soft lithography in biology and biochemistry. Annu Rev Biomed Eng, 2001, 3: 335-373

43 Xia Y N, Whitesides G M. Soft lithography. Annu Rev Mater Sci, 1998, 28: 153-184

44 Xia Y N, Whitesides G M. Soft lithography. Angew Chem Int Ed, 1998, 37: 551-575

45 Ostuni E, Chen C S, Ingber D E, et al. Selective deposition of proteins and cells in arrays of microwells. Langmuir, 2001, 17: 2828-2834

46 Ostuni E, Yan L, Whitesides G M. The interaction of proteins and cells with self-assembled monolayers of alkanethiolates on gold and silver. Colloids Surf B, 1999, 15: 3-30

47 Kane R S, Takayama S, Ostuni E, et al. Patterning proteins and cells using soft lithography. Biomaterials, 1999, 20: 2363-2376

48 Khademhosseini A, Jon S, Suh K Y, et al. Direct Patterning of protein- and cell-resistant polymeric monolayers and microstructures. Adv Mater, 2003, 15: 1995-2000

49 Suh K Y, Khademhosseini A, Yang J M, et al. Soft lithographic patterning of hyaluronic acid on hydrophilic substrates using molding and printing. Adv Mater, 2004, 16: 584-588

50 Walker G M, Zeringue H C, Beebe D J. Microenvironment design considerations for cellular scale studies. Lab Chip, 2004, 4: 91-97

51 Inaba $\mathrm{R}$, Khademhosseini A, Suzuki $\mathrm{H}$, et al. Electrochemical desorption of self-assembled monolayers for engineering cellular tissues. Biomaterials, 2009, 30: 3573-3579

52 Kato-Negishi M, Tsuda Y, Onoe H, et al. A neurospheroid network-stamping method for neural transplantation to the brain. Biomaterials, 2010, 31: 8939-8945

53 Schulze S, Huang G, Krause M, et al. Morphological Differentiation of Neurons on Microtopographic Substrates Fabricated by Rolled-Up Nanotechnology. Adv Eng Mater, 2010, 12: B558-B564

54 Yu M, Huang Y, Ballweg J, et al. Semiconductor Nanomembrane Tubes: Three-Dimensional Confinement for Controlled Neurite Outgrowth. ACS Nano, 2011, 5: 2447-2457

55 Yuan B, Jin Y, Sun Y, et al. A strategy for depositing different types of cells in three dimensions to mimic tubular structures in tissues. Adv Mater, 2012, 24: 890-896

56 Takeichi M. Cadherins-a molecular family important in selective cell-cell adhesion. Annu Rev Biochem, 1990, 59: 237-252

57 Hynes R O, Lander A D. Contact and adhesive specificities in the associations, migrations, and targeting of cells and axons. Cell, 1992, 68: 303-322

58 Kellam B, De Bank P A, Shakesheff K M. Chemical modification of mammalian cell surfaces. Chem Soc Rev, 2003, 32: 327-337

59 Stephan M T, Irvine D J. Enhancing cell therapies from the outside in: Cell surface engineering using synthetic nanomaterials. Nano Today, 2011, 6: 309-325

60 Sarkar D, Vemula P K, Teo G S L, et al. Chemical engineering of mesenchymal stem cells to induce a cell rolling response. Bioconjugate Chem, 2008, 19: 2105-2109

61 Krishnamachari Y, Pearce M E, Salem A K. Self-assembly of cell-microparticle hybrids. Adv Mater, 2008, 20: 989-993

62 Kojima N, Takeuchi S, Sakai Y. Establishment of self-organization system in rapidly formed multicellular heterospheroids. Biomaterials, 2011, 32: 6059-6067

63 Vermesh U, Vermesh O, Wang J, et al. High-density, multiplexed patterning of cells at single-cell resolution for tissue engineering and other applications. Angew Chem Int Ed, 2011, 50: 7378-7380

64 Stephan M T, Moon J J, Um S H, et al. Therapeutic cell engineering with surface-conjugated synthetic nanoparticles. Nat Med, 2010, 16: 1035-1041

65 De Bank P A, Kellam B, Kendall D A, et al. Surface engineering of living myoblasts via selective periodate oxidation. Biotechnol Bioeng, 2003, 81: 800-808 
66 De Bank P A, Hou Q, Warner R M, et al. Accelerated formation of multicellular 3-D structures by cell-to-cell cross-linking. Biotechnol Bioeng, 2007, 97: 1617-1625

67 White S H, von Heijne G. Transmembrane helices before, during, and after insertion. Curr Opin Struct Biol, 2005, 15: 378-386

68 Teramura Y, Iwata H. Islet encapsulation with living cells for improvement of biocompatibility. Biomaterials, 2009, 30: 2270-2275

69 Teramura Y, Minh L N, Kawamoto T, et al. Microencapsulation of islets with living cells using polydna-peg-lipid conjugate. Bioconjugate Chem, 2010, 21: 792-796

70 Teramura Y, Chen H, Kawamoto T, et al. Control of cell attachment through polyDNA hybridization. Biomaterials, 2010, 31: 2229-2235

71 Dutta D, Pulsipher A, Luo W, et al. Synthetic chemoselective rewiring of cell surfaces: generation of three-dimensional tissue structures. J Am Chem Soc, 2011, 133: 8704-8713

72 Kayser H, Zeitler R, Kannicht C, et al. Biosynthesis of a nonphysiological sialic-acid in different rat organs, using n-propanoyl-d-hexosamines as precursors. J Biol Chem, 1992, 267: 16934-16938

73 Prescher J A, Dube D H, Bertozzi C R. Chemical remodelling of cell surfaces in living animals. Nature, 2004, 430: 873-877

74 Dube D H, Bertozzi C R. Metabolic oligosaccharide engineering as a tool for glycobiology. Curr Opin Chem Biol, 2003, 7: 616-625

75 Mahal L K, Yarema K J, Bertozzi C R. Engineering chemical reactivity on cell surfaces through oligosaccharide biosynthesis. Science, 1997, 276: 1125-1128

76 Iwasaki Y, Tabata E, Kurita K, et al. Selective cell attachment to a biomimetic polymer surface through the recognition of cell-surface tags. Bioconjugate Chem, 2005, 16: 567-575

77 Nauman D A, Bertozzi C R. Kinetic parameters for small-molecule drug delivery by covalent cell surface targeting. Biochim Biophys Acta Gen Subj, 2001, 1568: 147-154

78 Lee J H, Baker T J, Mahal L K, et al. Engineering novel cell surface receptors for virus-mediated gene transfer. J Biol Chem, 1999, 274: 21878-21884

79 Sampathkumar S G, Li A V, Jones M B, et al. Metabolic installation of thiols into sialic acid modulates adhesion and stem cell biology. Nat Chem Biol, 2006, 2: 149-152

80 Gartner Z J, Bertozzi C R. Programmed assembly of 3-dimensional microtissues with defined cellular connectivity. Proc Natl Acad Sci USA, 2009, 106: 4606-4610

81 Howarth M, Takao K, Hayashi Y, et al. Targeting quantum dots to surface proteins in living cells with biotin ligase. Proc Natl Acad Sci USA, 2005, 102: 7583-7588

82 Chen I, Howarth M, Lin W, et al. Site-specific labeling of cell surface proteins with biophysical probes using biotin ligase. Nat Methods, 2005, 2: 99-104

83 Rajagopalan P, Shen C J, Berthiaume F, et al. Polyelectrolyte nano-scaffolds for the design of layered cellular Architectures. Tissue Eng, 2006, 12: 1553-1563

84 Fischer D, Li Y X, Ahlemeyer B, et al. In vitro cytotoxicity testing of polycations: influence of polymer structure on cell viability and hemolysis. Biomaterials, 2003, 24: 1121-1131

85 Chanana M, Gliozzi A, Diaspro A, et al. Interaction of polyelectrolytes and their composites with living cells. Nano Lett, 2005, 5: 2605-2612

86 Chen W, Zarnitsyna V, Sarangapani K, et al. Measuring receptor-ligand binding kinetics on cell surfaces: from adhesion frequency to thermal fluctuation methods. Cell Mol Bioeng, 2008, 1: 276-288

87 Kickhoefer V A, Han M, Raval-Fernandes S, et al. Targeting vault nanoparticles to specific cell surface receptors. ACS Nano, 2008, 3: 27-36

88 Swiston A J, Cheng C, Um S H, et al. Surface functionalization of living cells with multilayer patches. Nano Lett, 2008, 8: 4446-4453

89 Chen X, Tam U C, Czlapinski J L, et al. Interfacing carbon nanotubes with living cells. J Am Chem Soc, 2006, 128: 6292-6293

90 Yamada K M. Cell surface interactions with extracellular materials. Annu Rev Biochem, 1983, 52: 761-799

91 Ruoslahti E, Pierschbacher M. New perspectives in cell adhesion: RGD and integrins. Science, 1987, 238: 491-497

92 Matsusaki M, Kadowaki K, Nakahara Y, et al. Fabrication of cellular multilayers with nanometer-sized extracellular matrix films. Angew Chem Int Ed, 2007, 46: 4689-4692

93 Nishiguchi A, Yoshida H, Matsusaki M, et al. Rapid construction of three-dimensional multilayered tissues with endothelial tube networks by the cell-accumulation technique. Adv Mater, 2011, 23: 3506-3510

Open Access This article is distributed under the terms of the Creative Commons Attribution License which permits any use, distribution, and reproduction in any medium, provided the original author(s) and source are credited. 